\title{
Effects of 3d transition metal impurities and vacancy defects on electronic and magnetic properties of pentagonal Pd2S4 : Competition between exchange splitting and crystal fields
}

\section{Mojtaba gholami}

University Campus2, University of Guilan

\section{Zahra Golsanamlou}

University of Guilan

Hamid Rahimpour soleimani ( $\nabla$ rahimpour@guilan.ac.ir)

University of Guilan

\section{Research Article}

Keywords: Pd2S4 monolayer, Transition-metal dichalcogenides, Vacancy defects, Crystal field, Exchange splitting

Posted Date: February 23rd, 2022

DOI: https://doi.org/10.21203/rs.3.rs-1353589/v1

License: (c) (1) This work is licensed under a Creative Commons Attribution 4.0 International License.

Read Full License 


\title{
Effects of 3d transition metal impurities and vacancy defects on electronic and magnetic properties of pentagonal $\mathrm{Pd}_{2} \mathrm{~S}_{4}$ : Competition between exchange splitting and crystal fields
}

\author{
Mojtaba gholami ${ }^{1.2}$, Z. Golsanamlou ${ }^{2}$, H. Rahimpour Soleimani ${ }^{1,2} *$ \\ ${ }^{1}$ Department of physics, University Campus2, University of Guilan, Rasht, Iran. \\ ${ }^{2}$ Computational Nanophysics Laboratory (CNL), Department of Physics, University of Guilan, P. O. Box \\ 41335-1914, Rasht, Iran \\ * Corresponding authors: H. Rahimpour Soleimani (Rahimpour @guilan.ac.ir)
}

\section{Abstract}

In this paper, we first investigate the electronic properties of the two-dimensional structure of dichalcogenide $\mathrm{Pd}_{2} \mathrm{~S}_{4}$. These properties strongly depend on the crystal field splitting which can change by atomic vacancies ( $S$ and $P d$ vacancies). The main purpose of the present paper is to create remarkable magnetic properties in the system by adding $3 \mathrm{~d}$ transition metal atoms where the presence of $\mathrm{Mn}, \mathrm{Cr}$, and Fe creates the exchange interaction in the system as well as change in the crystal field. The created magnetic properties strongly depend on the competition between exchange interaction and crystal field to separate the levels of $d$ orbitals. In addition, the presence of the transition metals in the structures with $\mathrm{S}$ and Pd vacancy has been investigated carefully. The calculations demonstrate that we can achieve an extensive range of magnetic moment up to $3.131 \mu \_B$. The maximum one is obtained in the presence of $\mathrm{Mn}$ and absence of sulfur while some of the doped structures does not have magnetic moment. Our results show that Pd vacancy in the presence of $\mathrm{Cr}, \mathrm{Mn}$ and $\mathrm{Fe}$ metals increases the magnetic property of the $\mathbf{P d}_{2} \mathrm{~S}_{4}$ structure. The extensiveness and variety of the obtained properties can be used for different magnetic and non-magnetic applications.

Keyword: $\mathrm{Pd}_{2} \mathrm{~S}_{4}$ monolayer, Transition-metal dichalcogenides, Vacancy defects, Crystal field, Exchange splitting

\section{Introduction}

The two-dimensional (2D) Transition-metal dichalcogenides (TMDs) are among the significant 2D structures vastly studied and analyzed recently. Most of these materials have unique 2D characteristics that have never been observed in three-dimensional structures ${ }^{1-3}$. TMDs mostly form an essential group of multi-layer materials with a general formula of $\mathrm{MX}_{2}$. In this formula, $\mathrm{M}$ and $\mathrm{X}$ represent a transition material and a chalcogenide element, respectively ${ }^{4}$. These materials are comprised of $2 \mathrm{D}$ layers. Each layer includes three chalcogenide atoms, and in the middle of each atom, there is a metal element. There also exist weak covalent bonds among them, enabling the formation of a layer. TMC structures have unique chemical and impressive physical characteristics due to their chemical bond conditions ${ }^{5}$. 
There are numerous investigations conducted on TMC materials magnetization ${ }^{6-15}$. Most of these materials do not hold magnetic features in their initial synthesized form, despite their remarkable electronic and mechanical properties. Since many novel applications can be achieved by adding magnetic characteristics to these 2D structures, extensive studies and research are performed in this field. The regular methods to study the magnetic properties are based on the topology method, applying strain forces, vacuuming, and defect generation. In addition, one of the most significant magnetization methods is doping the material with transition elements of different periods of the periodic table. Some of the works performed in this area are noted in the following ${ }^{16-28}$. Octa et al. ${ }^{17}$ created the $\mathrm{T}$ and $\mathrm{H}$-phase conditions on an extensive range of TMCs and investigated the magnetization conditions on the structures. They revealed that VX2 (where X could be $\mathrm{O}, \mathrm{S}, \mathrm{Se}$, or Te) is magnetized at each of the two phases, while NiX2 is not. They also demonstrated that the magnetization of ScX2 depends on the phase type. Wang et al. studied the 2D MoS2 material's magnetic characteristics by creating defects and vacuuming ${ }^{21}$. They observed that magnetizing the structure could not be achieved only by vacuuming. Therefore, they combined the vacuuming method by doping with materials like $\mathrm{Mn}, \mathrm{Fe}$, and $\mathrm{Co}$ and observed the magnetic properties of the system clearly. Applying strain is one of the other magnetization methods. The work by Liu et al. can be noted in this regard ${ }^{22}$. They revealed that gradual increase of strain magnetizes the 2D materials of $\mathrm{CrSe} 2$ and CsTe2 and even generates ferromagnetic and antiferromagnetic properties in the structures. As mentioned earlier, including defects is another 2D TMC materials' magnetization technique. Defect creation in a $\mathrm{MX}_{2}$ structure can be a consequence of eliminating the structure's $\mathrm{X}$ or $\mathrm{M}$ atoms. Avsar et al. ${ }^{23}$ studied the magnetization of a PtSe2 structure by removing Se and Pt atoms. Their results revealed that a Pt vacancy increases the magnetization of the system by $1.2 \mu \mathrm{B}$. In contrast, a Se vacancy does not produce any magnetization effects. Yandong Ma et al. investigated the MoTe2 and MoSe2 structures by applying defects and removing Mo atoms ${ }^{24}$. Their study demonstrated that only MoSe2 structures have spin polarization and magnetic properties. In another work, Nan Gao et al. observed that MoSe2 structures do not acquire magnetic characteristics by applying $S$ vacancy defects ${ }^{25}$. One of the common and accessible approaches to magnetize the structures is the 2D TMC material doping method. There have been numerous studies in this field. Some of these works are noted here. The majority of dopant materials are transition metal elements of the periodic table. Zhang et al. doped the 2D metal ( $\mathrm{CrS2}$ ) with transition elements and some alkaline earth elements like $\mathrm{Ca}$ and demonstrated that the dopants except vanadium manage to magnetize the structure $\mathrm{e}^{26}$. They deduced that the acquired magnetization of MoS2, when doped by F, N, B, and H elements, is similar and has a value of $1.0 \mu \mathrm{B}$, while the magnetic influence of $\mathrm{Co}, \mathrm{Fe}, \mathrm{Mn}, \mathrm{Cr}$, and $\mathrm{V}$ elements on MoS2 are different ${ }^{27}$. Alexander et al. studied the effects of doping MoS2 materials with $3 \mathrm{~d}$ transition metals and resulted that $\mathrm{Fe}, \mathrm{Mn}$, and $\mathrm{Co}$ elements provide the maximum magnetic intensities in their doped structures ${ }^{28}$.

By doping ZrS2 with transition elements and alkaline metals, Bayshan Yang et al. demonstrated that $\mathrm{Mn}$ and $\mathrm{Cr}$ elements have the greatest magnetic influence, while $\mathrm{Ni}$ and Ti have no role in the system's magnetization $^{29}$. In addition, Hashemi and his group performed a new examination on doping of the 2D structure of WSe2 using $4 \mathrm{~d}$ elements and proved magnetization using $\mathrm{Zr}$, Nb, Mo, and Tc. However, they did not achieve any magnetic effect in materials doped by $\mathrm{Y}, \mathrm{Ru}, \mathrm{Rh}$, and Pd elements ${ }^{30}$.

$\mathrm{Pd}_{2} \mathrm{~S}_{4}$ is one of the fascinating chalcogenide structures of transition elements. It is composed of a palladium atom and two sulfur atoms. This structure is a member of $2 \mathrm{D}$ pentagonal $\mathrm{TM}_{2} \mathrm{X}_{4}$ materials (where TM could be transition metal and X could be S, Se, or Te) despite the other transition elements' chalcogenides that are quadrangular or hexagonal. Therefore, it is one of the $2 \mathrm{D}$ chalcogenide materials that could be viewed as an intrinsic semiconductor ${ }^{31}$. It transforms easily from a semiconductor to a conductor under a particular metamorphosis (e.g., stress) and turns into sheet form from a bulk state. This transformation capability gives the material some exciting electronic and photonic characteristics. In 
fact, it has unique anisotropic features with a gap energy of almost $1.2 \mathrm{eV}$. In other words, $\mathrm{Pd}_{2} \mathrm{~S}_{4}$ is a semiconductor from an electric conduction viewpoint and has an indirect bandgap. However, it has a $1.1 \mathrm{eV}$ gap in 1T-phase mode and a zero-gap in bulk mode. Moreover, the two-layer structure of $\mathrm{Pd}_{2} \mathrm{~S}_{4}$ has a gap of $0.952 \mathrm{eV}^{32-35}$. There has also been some research on the edges of the $\mathrm{Pd}_{2} \mathrm{~S}_{4}$ structure. This structure has different electric properties in nanoribbon mode compared with the bulk mode, so that exhibits a metal-like behavior in zigzag mode and semiconductor behavior in armchair mode. Although there are extensive research articles on $\mathrm{Pd}_{2} \mathrm{~S}_{4}$ in the fields of electronics and magnetism, there are not sufficient thorough studies on doping these materials ${ }^{36-39}$.

In this paper, the magnetic features of $\mathrm{Pd}_{2} \mathrm{~S}_{4}$ structure are studied through doping with $3 \mathrm{~d}$ transition metals ( $\mathrm{Sc}, \mathrm{Ti}, \mathrm{V}, \mathrm{Cr}, \mathrm{Mn}, \mathrm{Fe}, \mathrm{Co}, \mathrm{Ni}, \mathrm{Cu}$, and $\mathrm{Zn}$ ) and creating a defect by removing a $\mathrm{Pd}$ atom and $\mathrm{S}$ atom. To the best of our knowledge, there is no published research article on doped $\mathrm{Pd}_{2} \mathrm{~S}_{4}$ structure by $3 \mathrm{~d}$ transition metal in the presence of Pd and S vacancies. Firstly, the spin behaviors of the orbitals of the valance bands of $S$ and $\mathrm{Pd}$ atoms are analyzed. Furthermore, their behaviors at the valance and conduction bands boundary are studied without considering the doping and vacancy effects on the structure. Then, the impact of $\mathrm{Pd}$ and $\mathrm{S}$ vacancies are examined, and the achieved results are analyzed. Finally, to conduct a more comprehensive study, situations with and without vacancy are considered in the presence of $3 \mathrm{~d}$ metals. In addition, the effects of $3 d$ doping and defect on the gap energy value are investigated. The simultaneous evaluation of the effects of doping and defect on the material is one of the considerable aspects of this work. It is demonstrated that the doping has more influence on magnetization than the atomic vacancies. In other words, doping with some elements leads to magnetization, while doping with some other elements causes no magnetic effects. Moreover, both doping and vacancies have significant roles in reducing the gap energy and changing its type.

\section{Methods}

Our Density functional theory (DFT) calculations based on the Perdew, Burke and Ernzerhof (PBE) ${ }^{40}$ variant of the exchange-correlation functional and the projector augmented wave (PAW) pseudopotentials, ${ }^{41}$ as implemented in Siesta ${ }^{42}$ has been employed. The non-relativistic version of SGGA is used for correlation-equilibrium potential and GGA is used for non-polarization mode. The set of basic states used for the valances of TM-3d, S and Pd atoms are (3s 3p 4s 3d), (3s 3p), and (4s 3d 4p 4d), respectively. To find the equilibrium point in the calculations and the accuracy in calculating the atomic force, the relaxation value reached less than $0.05 \mathrm{eV} / \mathrm{A}^{\circ}$ and also the accuracy of the calculations $10^{-5} \mathrm{eV}$ has been considered. in evaluation related to magnetic state and the relaxation, The Brillouin zone was sampled with a $40 \times 40 \times 1$ and $14 \times 14 \times 1$, respectively. Our supercell used with dimensions of $4 \times 4 \times 1$ has $22 \mathrm{Pd}$ atoms and $48 \mathrm{~S}$ atoms as one TM. The bond lengths of Pd-S and TM-S atoms after optimization are set to be $2.358 \mathrm{~A}^{\circ}$ and $2.332 \mathrm{~A}^{\circ}$, respectively. To study the electric and magnetic characteristics of TMdoped system, the LBFGS-optimized structures were used. besides Tolerable force and pressure are assumed to be $0.05 \mathrm{eV} / \mathrm{A}^{\circ}$ and $0.2 \mathrm{GPa}$, respectively. 


\section{Results}

\section{Electronic properties of monolayer $\mathrm{Pd}_{2} \mathrm{~S}_{4}$}

The monolayer $\mathrm{Pd}_{2} \mathrm{~S}_{4}$ belongs to a simple orthorhombic crystal lattice structure with space group $\mathrm{Pbca}$ (P2_1/c) and point group $(2 / \mathrm{m})$. These compounds are composed of $\mathrm{S}-\mathrm{Pd}-\mathrm{S}-\mathrm{S}-\mathrm{Pd}-\mathrm{S}$ planes where $\mathrm{Pd}-\mathrm{S}$ and $\mathrm{S}-\mathrm{S}$ are ionic and covalent bonds, respectively. Fig. 1 illustrates the top view (p) and side view (q) of a $2 \times 2$ supercell of $\mathrm{Pd}_{2} \mathrm{~S}_{4}$ monolayer. As it can be seen, the positioning of palladium atoms in this structure is so that every palladium atom is in contact with four sulfur atoms. Each pentagonal ring of $\mathrm{Pd}_{2} \mathrm{~S}_{4}$ includes four $\mathrm{Pd}-\mathrm{S}$ bonds and an $\mathrm{S}-\mathrm{S}$ bond. After optimizing the system, computations indicate that the lattice constants and bond length between a palladium atom and nearest sulfur atoms is $a=5.4833, b=5.4833, c=16.3359$, and $d_{P d-s}=3.433 \AA$. Also, the covalent bond $\mathrm{S}-\mathrm{S}$ has a length of $d_{s-s}=2.12 \AA$. Concerning the crucial importance of valence electrons in establishing the electronic and magnetic properties, atomic orbitals $\operatorname{Pd}\left(5 \mathrm{~s}^{0}, 4 \mathrm{~d}^{10}\right)$ and $\mathrm{S}\left(3 \mathrm{~s}^{2}, 3 \mathrm{p}^{4}\right)$ play an essential role in structural hybridization. Fig. 1(a) shows the band structure and density of states (DOS) of orbitals in the valence band of sulfur $\mathrm{S}(3 \mathrm{~s}, 3 \mathrm{p})$ and palladium $\mathrm{Pd}(5 \mathrm{~s}, 4 \mathrm{~d})$. As shown in Fig. 1(a), the $d$ orbitals of the palladium and $p$ orbitals of the sulfur have significant contributions in structure formation. These valence and conduction bands' orbitals exhibit a complete overlap and proper interactions. The two $3 p$ and $4 \mathrm{~d}$ orbitals in $\mathrm{S}$ and $\mathrm{Pd}$ atoms, respectively, have more contribution in the valence band maximum (VBM) and conduction band minimum (CBM). These orbitals are the linking bridges between ligands and metals in $\mathrm{Pd}_{2} \mathrm{~S}_{4}$. Fig.1(a) also reveals that 3 s orbitals in $\mathrm{S}$ atoms and $5 \mathrm{~s}$ orbitals in $\mathrm{Pd}$ atoms have the lowest DOS in valence and conduction bands and cannot play an essential role in the electronic structure. In the following, we generally concentrate our investigation on $3 p$ orbitals in S atoms and $4 d$ orbitals in Pd atoms. In a straightforward and approximate embodiment, it can be stated that when the $\mathrm{S}$ atom takes place in a monolayer structure, it can obtain two electrons from adjacent $\mathrm{Pd}$ atoms due to the higher electronegativity than $\mathrm{Pd}$. Because of locating next to four $\mathrm{S}$ atoms, the $\mathrm{Pd}$ atom is converted to $\mathrm{Pd}^{4+}$ after hybridization. The five orbitals $\mathrm{d}_{\mathrm{xy}}, \mathrm{d}_{\mathrm{yz}}, \mathrm{d}_{\mathrm{zx}}, \mathrm{d}_{\mathrm{z}^{2}}, \mathrm{~d}_{\mathrm{x}^{2}-\mathrm{y}^{2}}$ of the $P d$ atom are split under the effect of the crystal field. The $4 \mathrm{~d}$ orbitals are greater in extent compared to $3 \mathrm{~d}$ ones. In addition, the electronic and magnetic properties of the system are significantly dependent on how orbitals are filled close to the Fermi level. Considering the evaluations near the Fermi level, the splitting mechanism is demonstrated in Fig.1(ae) regarding the results obtained from the DOS diagram of $4 \mathrm{~d}$ orbitals of the Pd atom. Among five orbitals

$\mathrm{d}_{\mathrm{xy}}, \mathrm{d}_{\mathrm{yz}}, \mathrm{d}_{\mathrm{zx}}, \mathrm{d}_{\mathrm{z}^{2}}, \mathrm{~d}_{\mathrm{x}^{2}-\mathrm{y}^{2}}$ (Fig.1(c-e)) of Pd atom, $\mathrm{d}_{\mathrm{xy}}$ and $\mathrm{d}_{\mathrm{x}^{2}-\mathrm{y}^{2}}$ (Fig.1(e)) have the highest number of vacancy states. Three remaining orbitals have no DOS at energy levels higher than Fermi energy. Based on these data, how orbitals are positioned and how they are filled can be predicted regarding to the crystal field and experimental results. Five orbitals of palladium atom in the energy range of -2 to $+2 \mathrm{eV}$ are almost placed in four different levels. As shown in Fig.1(r), orbitals can be filled in two ways. In the first state which is high-spin configuration, all spitted d-orbitals almost have electrons. 

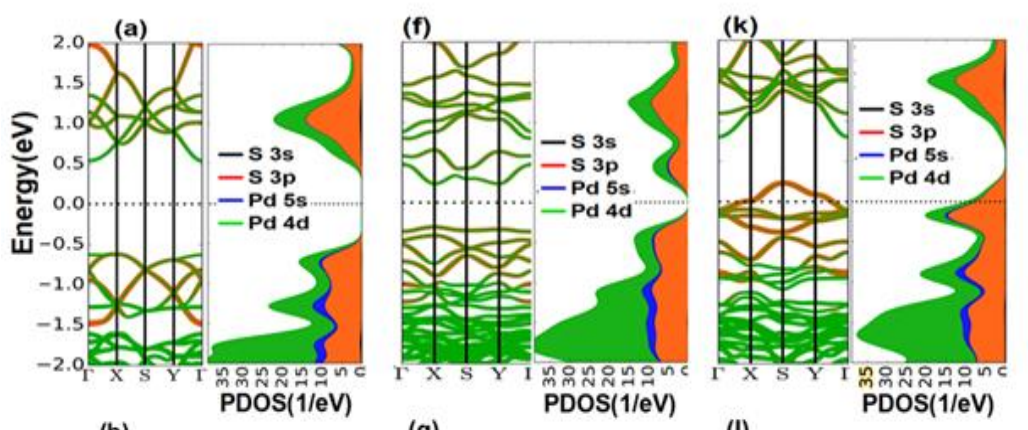

(p)

(q)
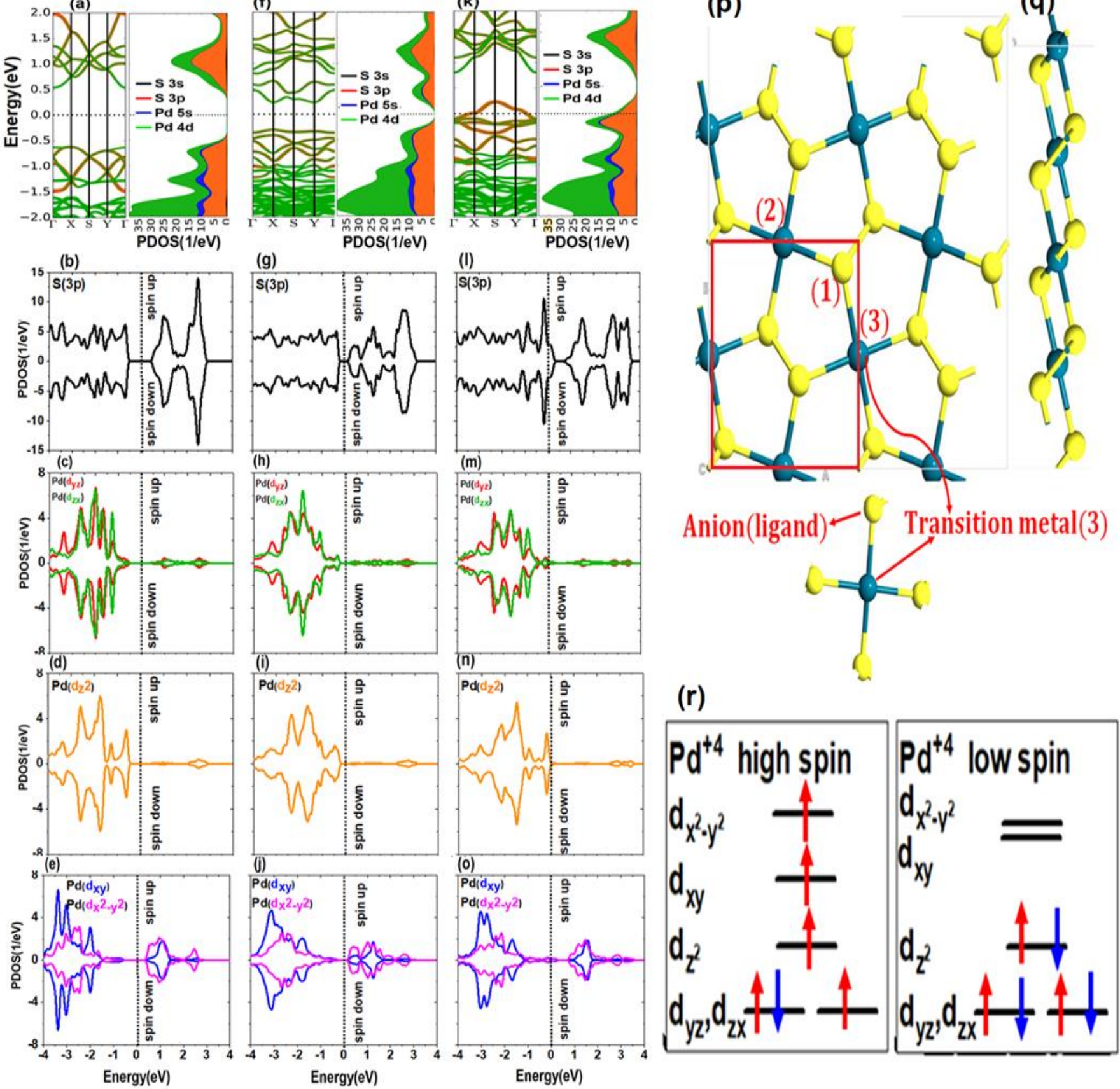

(r)
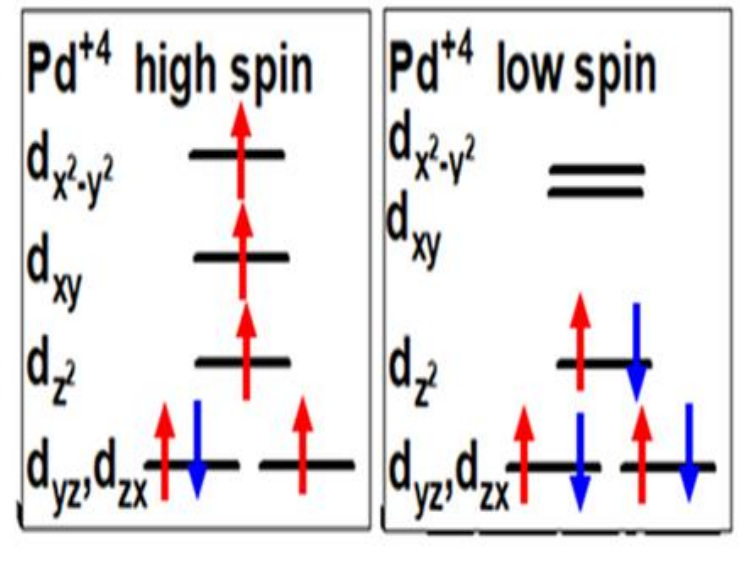

Figure 1. Band structure and density of states for $3 p$ orbitals of $S$ atom and $4 d$ orbitals of $P d$ atom in $\mathrm{Pd}_{2} \mathrm{~S}_{4}$ structure: (a-e) without vacancy defect, $(\mathrm{f}-\mathrm{j})$ arising from defects in $\mathrm{S}$ atoms, $(\mathrm{k}-\mathrm{o})$ arising from defects in $P d$ atoms, (p) top view of $\mathrm{Pd}_{2} \mathrm{~S}_{4}$ structure with the position of vacancy defect for $\mathrm{S}$ (No. 1 ) and $\mathrm{Pd}$ (No. 2) atoms, (q) side view of $\mathrm{Pd}_{2} \mathrm{~S}_{4}$ structure, and $(r)$ electrons located in $4 \mathrm{~d}$ orbitals of $\mathrm{Pd} 4+$ cation in strong and weak crystal fields. 
In other state or low-spin configuration, $\mathrm{d}_{\mathrm{yz}}$ and $\mathrm{d}_{\mathrm{zx}}$ (Fig.1(c)) are degenerate orbitals. These are the lowest $d$ orbitals in terms of energy level for both high and low spins states and are fully occupied by low spin states. The $d_{z^{2}}$ (Fig.1(d)) orbital is also full and has an energy level higher than $d_{y z}$ and $d_{z x}$ (Fig.1(c)). However, $\mathrm{d}_{\mathrm{xy}}$ and $\mathrm{d}_{\mathrm{x}^{2}-\mathrm{y}^{2}}$ (Fig.1(e))are vacant and have much higher energy levels. Being in one of these two states depends on the strength and weakness of the crystal field and the separation of d-orbital energy levels. Since the $\mathrm{Pd}^{4+}$ structure includes $d$ orbitals, which are higher in energy with respect to $\mathrm{s}$ and $p$ orbitals, it well overlaps with $p$ orbitals of adjacent ligand $\mathrm{S}^{-2}$ in $\left[\mathrm{Pd}_{2} \mathrm{~S}_{4}\right]^{-2}$. As a result, because of $\mathrm{pd}$ hybridization between metallic and ligand orbitals, a strong field is created causing $d$ orbitals of palladium to be further split. According to Fig. 1(r), if the field is weak, the distance between d-orbital energy levels is less and more individual electrons occupy the levels, which results in the paramagnetic properties of palladium (high-spin). Also, if the field is strong, six electrons of $\mathrm{Pd}^{4+}$ are located pairwise in $\mathrm{d}_{\mathrm{yz}}, \mathrm{d}_{\mathrm{zx}}$ (Fig.1(c)), and $\mathrm{d}_{\mathrm{z}^{2}}$ (Fig.1(d)) orbitals in lower energies and higher energy orbitals ( $\mathrm{d}_{\mathrm{xy}}$ and $\mathrm{d}_{\mathrm{x}^{2}-\mathrm{y}^{2}}($ Fig.1(e))) are almost vacant (low-spin). Therefore, high and low spins of $\mathrm{d}_{\mathrm{yz}}, \mathrm{d}_{\mathrm{zx}}$ (Fig.1(c)) and $\mathrm{d}_{\mathrm{z}^{2}}$ (Fig.1(d)) orbitals completely neutralize each other, and the structure will have no magnetic properties. Obtained results show that $\mathrm{Pd}_{2} \mathrm{~S}_{4}$ has no magnetic property, which is consistent with experimental results ${ }^{43-44}$. Thus, in the square planar form, the $\mathrm{d}$ orbitals of $\mathrm{Pd}$ are split so that four single electrons prefer to be paired and carry the pairing energy than transferring to higher energy orbitals which are $d_{x y}$ and $d_{x^{2}-y^{2}}$ (Fig.1(e)) The $\mathrm{p}_{x}, \mathrm{p}_{\mathrm{y}}$, and $\mathrm{p}_{\mathrm{z}}$ (Fig.1(b))orbitals of the $\mathrm{S}$ atom experience no significant change in crystal field due to their symmetric geometry and place in the same energy level. The obtained results indicate that $\operatorname{Pd}_{2} \mathrm{~S}_{4}$ monolayer is a nonmagnetic semiconductor with an indirect gap energy of $1.2 \mathrm{eV}$, which does not cross any part of the Fermi level because of its quite symmetrical and identical high-spin and low-spin DOS (see Fig.1(b-e)). In the square planar structure, electrons of $\mathrm{Pd}^{4+}$ fill the energy levels from bottom to top. Given that six high-spin and low-spin electrons have filled three first levels, it can be concluded that no spin magnetization occurs in the pure $\mathrm{Pd}_{2} \mathrm{~S}_{4}$ system. It is clear from the comparison of $4 \mathrm{~d}$ orbitals of $\mathrm{Pd}$ that although $\mathrm{d}_{\mathrm{z}^{2}}$ (Fig.1(d)) has a more considerable contribution than $\mathrm{d}$ orbitals in a specific valence band in the range of $-1.5 \mathrm{eV}$ to $-0.25 \mathrm{eV}$, it has a minor contribution in the conduction band. The $\mathrm{d}_{\mathrm{xy}}$ and $\mathrm{d}_{\mathrm{x}^{2}-\mathrm{y}^{2}}$ (Fig.1(e)) orbitals of Pd devotes the highest contribution in the conduction band, but $\mathrm{d}_{\mathrm{yz}}, \mathrm{d}_{\mathrm{zx}}$ (Fig.1(c)), and $\mathrm{d}_{\mathrm{z}^{2}}$ (Fig.1(d)) play more critical role in the formation of valence band due to their lower energy level and higher DOS. In the following, we investigate the effect of removing S (1) nearest adjacent to the Pd atom, as shown in Fig.1(p). As can be seen, vacancy defect causes the two Pd atoms and one $\mathrm{S}$ atom to have a free or suspended band. After removing $\mathrm{S}$, two electrons of $\mathrm{Pd}$ which have constrained by $\mathrm{S}$ become free. Therefore, these electrons can create new levels in the gap energy region and decrease the gap energy. Fig.1(f-j) displays the band structure and DOS of monolayer $\mathrm{Pd}_{2} \mathrm{~S}_{4}$ with a vacancy, where the gap energy of the system decreases from $0.82 \mathrm{eV}$ to $1.2 \mathrm{eV}$ keeps the direct gap energy and semiconductor structure. The VBM does not change his place whereas CBM changes from $\Gamma$ to $X$. New states, which play the role of electron acceptors and donors and provide more space for the movement of electrons, are formed in the vicinity of the Fermi level. Under the new crystal field effect, splitting $d$ orbitals of Pd and $p$ orbitals of $\mathrm{S}$ experience no major difference relative to the pure state. As shown in Fig.1(h), the pair of degenerate orbitals, $\mathrm{d}_{\mathrm{yz}}$ and $\mathrm{d}_{\mathrm{zx}}$, exhibits approximately similar behavior. Also, the $\mathrm{d}_{\mathrm{xy}}$ and $\mathrm{d}_{\mathrm{x}^{2}-\mathrm{y}^{2}}$ (Fig.1(j)) orbitals have almost similar behavior, while the single orbital $\mathrm{d}_{\mathrm{z}^{2}}$ (Fig.1(i)) is different from others. The monolayer $\mathrm{Pd}_{2} \mathrm{~S}_{4}$ maintains its nonmagnetic property because the DOS diagram of high-spin 
and low-spin states is symmetrical, as shown in Fig.1(g-j). A Pd atom is eliminated from the studied structure for a detailed examination of the crystal field. According to Fig.1(p), by removing the Pd atom No. 2, the geometrical symmetry of the system changes and the first four neighboring $S$ atoms will have free bonds in the system. Hence, those four $\mathrm{S}$ atoms have a half-filled orbital, which the electrons of that orbitals do not involve in the bond, can play a critical role in changing the band structure. Then, new states are created within the gap energy and adjacent to the Fermi level. Also, the new crystal field does not significantly affect the splitting of $d$ orbitals of Pd compared to the pure state. Based on Fig. $1(\mathrm{~m})$, the two orbitals $\mathrm{d}_{\mathrm{yz}}$ and $\mathrm{d}_{\mathrm{zx}}$ show almost the same behavior and are degenerate. Also, $\mathrm{d}_{\mathrm{xy}}$ and $\mathrm{d}_{\mathrm{x}^{2}-\mathrm{y}^{2}}$ (Fig.1(0)) orbitals have almost consistent diagrams, while the single orbital $\mathrm{d}_{\mathrm{z}^{2}}$ (Fig. $1(n)$ ) is different from others. In the valence band, the role of orbitals $d_{z^{2}}\left(\right.$ Fig.1(n)), $d_{y z}$ and $d_{z x}\left(\right.$ Fig.1(m)) is more than $d_{x y}$ and $d_{x^{2}-y^{2}}$ (Fig.1(o)), while the inverse situation can be found in the conduction band. According to Fig. 1(i), removing a Pd atom notably affects the $\mathrm{p}_{\mathrm{x}}, \mathrm{p}_{\mathrm{y}}$, and $\mathrm{p}_{\mathrm{z}}$ orbitals of the $\mathrm{S}$ atom so that the Fermi level is deeply shifted toward the interior of orbitals and plays a significant role in the conduction band of the structure. In this case, the system retains its nonmagnetic property, because the d orbitals of Pd atom and the $p$ orbitals of $\mathrm{S}$ atom have symmetrical DOS diagrams for the high-spin and the low-spin states.

\section{Electronic properties of monolayer $\mathrm{Pd}_{2} \mathrm{~S}_{4}$ in the presence of $3 \mathrm{~d}$ groups}

To create the magnetic property in the structure, as shown in Fig. 1, one of the Pd atoms of $\mathrm{Pd}_{2} \mathrm{~S}_{4}$ are substituted with the metals of $3 \mathrm{~d}$ group in three different cases. In the first case, the doping occurs in the pure system while in other two cases, the doping is in the presence of $\mathrm{Pd}$ and $\mathrm{S}$ vacancies. To investigate the doped system, the energy of magnetic $\left(E_{s p}\right)$ and nonmagnetic states $\left(E_{n s p}\right)$ due to the doping is calculated and compared between the three cases. The difference between magnetic and nonmagnetic energies of the systems is determined by $\Delta \mathrm{E}_{\text {spin }}=\mathrm{E}_{\mathrm{sp}}-\mathrm{E}_{\mathrm{nsp}}$. The obtained results for each case are presented in Table S1 of Supplementary Information (SI). The magnetic moment of the doped atom is indicated in Fig.2. By examining of the obtained results, the effects of $3 d$ transition metals on the magnetization of $\mathrm{Pd}_{2} \mathrm{~S}_{4}$ in considered cases can be divided into three groups: the first includes $\mathrm{Mn}, \mathrm{Cr}$, and Fe atoms which has the maximum values of $\Delta \mathrm{E}_{\text {spin }}$ and magnetic moment. The second group includes $\mathrm{V}, \mathrm{Ti}$, and $\mathrm{Co}$ atoms with moderate values of $\Delta \mathrm{E}_{\text {spin }}$ and magnetic moment. Finally, the third one involves $\mathrm{Sc}, \mathrm{Ni}, \mathrm{Cu}$, and $\mathrm{Zn}$ atoms where $\Delta \mathrm{E}_{\text {spin }}$ is zero. To further evaluate, a metal is selected as the representation of each group, and its properties are discussed in more details where, $\mathrm{Mn}, \mathrm{V}$, and $\mathrm{Ni}$ are examined as the representations of first, second, and third groups, respectively. Because of the importance of magnetic properties, the first group and $\mathrm{Mn}$ atoms are further investigated in this paper. The magnetization (Bohr magneton) of each metal and contributions of first and second neighbors of $\mathrm{S}$ and Pd in magnetization are demonstrated in Fig. 2 for three mentioned cases. The outstanding point is that the magnetization for the electrons of $d$-orbital cannot just be explained based on the splitting of $d$ orbitals according to the square planar model of the pure case (see Fig. 1(r)). The reason is that the presence of $3 \mathrm{~d}$ metals results in a strong exchange interaction besides the crystal field. The $3 \mathrm{~d}$ orbitals of the impurity atoms have a minor spatial extension than $4 \mathrm{~d}$ orbitals of the $\mathrm{Pd}$ atoms and are mainly local. Therefore, in most cases, there is a competition between these two fields to separate the $3 \mathrm{~d}$-orbital energy levels of the impurity, which determines the magnetic properties and moments. The first group 
and its representation (i.e., $\mathrm{Mn}$ ) are examined initially. When the $\mathrm{Mn}$ with the last layer atomic configuration of $3 d^{5} 4 s^{2}$ is substituted instead of $\mathrm{Pd}$ atom in the structure, it must lose the same electrons as the palladium loses (i.e., four electrons).

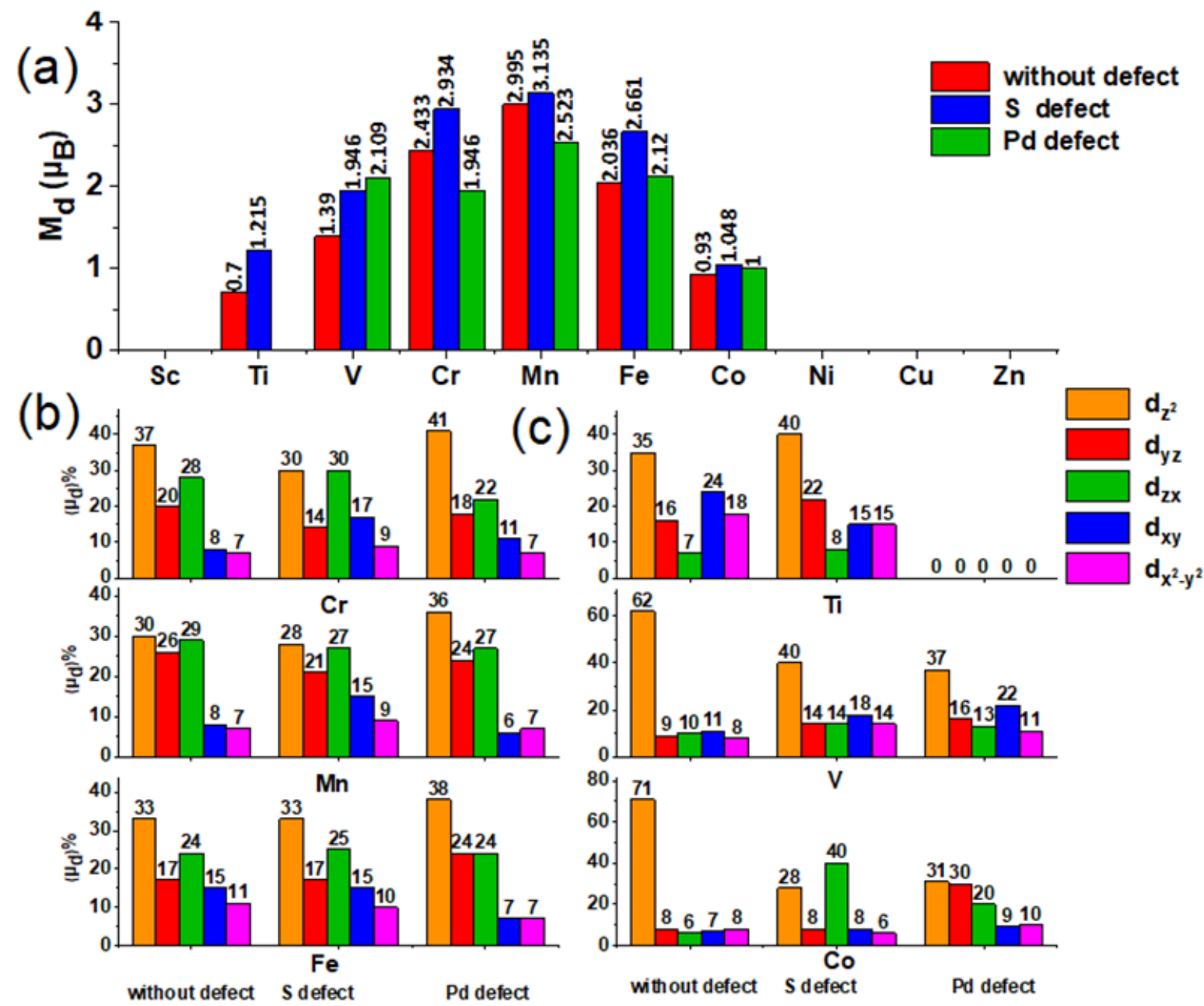

Figure 2. (a) Magnetic moment arising from doping of $\mathrm{Pd}_{2} \mathrm{~S}_{4}$ structure with $3 \mathrm{~d}$ transition metals with and without sulfur and palladium vacancies. (b) percentage of spin separation for five $3 \mathrm{~d}$ orbitals of the first group (high magnetization) with and without vacancies. (c) percentage of spin separation for five $3 \mathrm{~d}$ orbitals of the second group (medium magnetization) with and without vacancies.

According to Fig. 2(a), the magnetization intensity of $\mathrm{Mn}$ is $2.995 \mu_{B}$ in the absence of $\mathrm{S}$ and $\mathrm{Pd}$ vacances which is the maximum value among $3 \mathrm{~d}$ metals impurities. High-spin and low-spin electrons of $3 p$ orbitals of the $S$ atom are almost symmetrical in this case and play an insignificant role in the magnetic properties of the system. Fig. $3(a-f)$ shows the density states of $\mathrm{d}_{\mathrm{yz}}, \mathrm{d}_{\mathrm{zx}}, \mathrm{d}_{\mathrm{z}^{2}}, d_{x y}$, and $\mathrm{d}_{\mathrm{x}^{2}-\mathrm{y}^{2}}$ orbitals in the presence of $\mathrm{Mn}$ without vacancy defect. In this case, the system's spin separation or exchange interaction is strong. As shown in Fig. $3(d), d_{z^{2}}$ has the maximum locality and minimum bandwidth. The spin separation of $d_{z^{2}}$ is considerable and has profound effects on the magnetic moment of the system. The high spin and low spin of this orbital is occupied and unoccupied respectively. Also, the difference in energy levels of the two spin states is almost $4 \mathrm{eV}$. In addition to $d_{z^{2}}$ (Fig. 3(d)) orbital, the two $d_{y z}$ and $d_{z x}$ (Fig. 3(c)) orbitals 
of $\mathrm{Mn}$ are full in the high-spin state and vacant in the low-spin state. Hence, as shown in Fig. 2(a), regarding the portion of $3 \mathrm{~d}$ orbitals of $\mathrm{Mn}$ in the magnetic moment, it is observed that three orbitals of $\mathrm{d}_{\mathrm{z}}$, $\mathrm{d}_{\mathrm{yz}}$, and $\mathrm{d}_{\mathrm{zx}}$ involve in $30 \%, 26 \%$, and $29 \%$ (in total $85 \%$ ) of the obtained magnetic moment, respectively. Fig.S1 of SI presents the spin separation of these levels and deviation from the initial crystal field, schematically. According to DOS diagram in Fig. 3(e), the $d_{x y}$ and $d_{x^{2}-y^{2}}$ orbitals have larger spatial extents. The filling pattern of high-spin and low-spin states of these two orbitals indicates that they have no considerable effect on the system magnetization. Therefore, as $\mathrm{d}_{\mathrm{z}^{2}}, \mathrm{~d}_{\mathrm{yz}}$, and $\mathrm{d}_{\mathrm{zx}}$ are half-filled orbitals, the intensity of the magnetization for each high-spin state of these orbitals is almost $1 \mu_{B}$, which is $3 \mu_{B}$ overall. According to Fig.S2, the contribution of $3 \mathrm{~d}$ orbitals of $\mathrm{Mn}$ is effective in the band structure, and along with $3 \mathrm{p}$ orbitals of the $\mathrm{S}$ atom, it reduces the gap energy by $0.48 \mathrm{eV}$. For both $\mathrm{Mn}$ and $\mathrm{S}$ atoms, the high-spin orbitals in the valence band and low-spin orbitals in the conduction band are the main contributors in points VBM and CBM. These orbitals coincide with each other and are located at the $\Gamma$ point of the first Brillouin zone. Therefore, in the presence of $\mathrm{Mn}$, the gap energy reduces and changes to the direct type. In the last subfigure of Fig. 3(f), the spin density difference is presented for this case. Mn doping causes electrons of the first-neighbor $\mathrm{S}$ atom to have an opposite spin and be antiparallel relative to $\mathrm{Mn}$ electrons. The level of polarization becomes negligible by getting away from the impurity atom. Therefore, in the hybrid interaction of $d-p$ orbital, the electrons of $3 p$-orbital of the nearest $S$ atom and of $3 d$-orbital of the $\mathrm{Mn}$ atom have opposite spins.

To investigate the effect of atomic vacancy on the magnetic properties of the system in the presence of $\mathrm{TM}$, the sulfur atom which located in position 1 in Fig.1(p) is eliminated. As shown in Fig. 1(p), eliminating the $\mathrm{S}$ atom causes the TM (position No. 3), Pd (Position No.2), and S atoms (first neighbor to No.1) to have a free or suspended band. If one $\mathrm{S}$ atom is diminished from the structure, the $\mathrm{Mn}$ and $\mathrm{Pd}$ atoms lose one less electron relative to the previous state. Therefore, in the presence of $\mathrm{Mn}$ impurity, the metal cation $\mathrm{Mn}^{+3}$ can have more than three electrons in the $3 \mathrm{~d}$ level. According to Fig. 2(a), the magnetization intensity of Mn without S vacancy is almost $2.995 \mu_{B}$ and with $\mathrm{S}$ vacancy, it is $3.135 \mu_{B}$, indicating a $0.14 \mu_{B}$ increase relative to the former case. According to Fig. $3(\mathrm{~h})$, the spins of $3 p$-orbital of the $S$ atom are symmetrical and have no significant magnetic property. The $3 \mathrm{~d}$ orbitals have experienced some changes, of which the increased contribution of two planar orbitals $d_{x y}$ and $d_{x^{2}-y^{2}}$ (Fig. $3(k)$ ) to magnetization is one the important ones. This result is obtained from comparing data of magnetic contribution in Fig. 2(a) and results presented in Fig. 2(b) for the percentage contribution of Mn orbitals to the magnetic moment. The contributions of these orbitals, respectively, are $8 \%$ and $7 \%$ in the absence of the $S$ vacancy and $15 \%$ and $9 \%$ in the presence of vacancy. By removing the $S$ atom, the system has lower planar symmetry relative to the pure state. Hence, the contribution of two planar orbitals $d_{x y}$ and $d_{x^{2}-y^{2}}$ somewhat increases relative to the symmetric case. Based on data presented in Table S7, the gap energy is entirely disappeared, and the structure becomes conductive by removing the $S$ atom. Here, the role of $3 d$ and $3 p$ orbitals is also obvious so that VBM and CBM are almost tangential to the Fermi level at point $Y$, as shown in Figs. S2 and S3. It should be noted that, unlike the first case, low-spin orbitals in both valence and conduction bands play crucial roles. Albeit, in the valence zone, the role of $3 \mathrm{~d}$ orbital is of lower importance than that of $3 p$ orbital. 

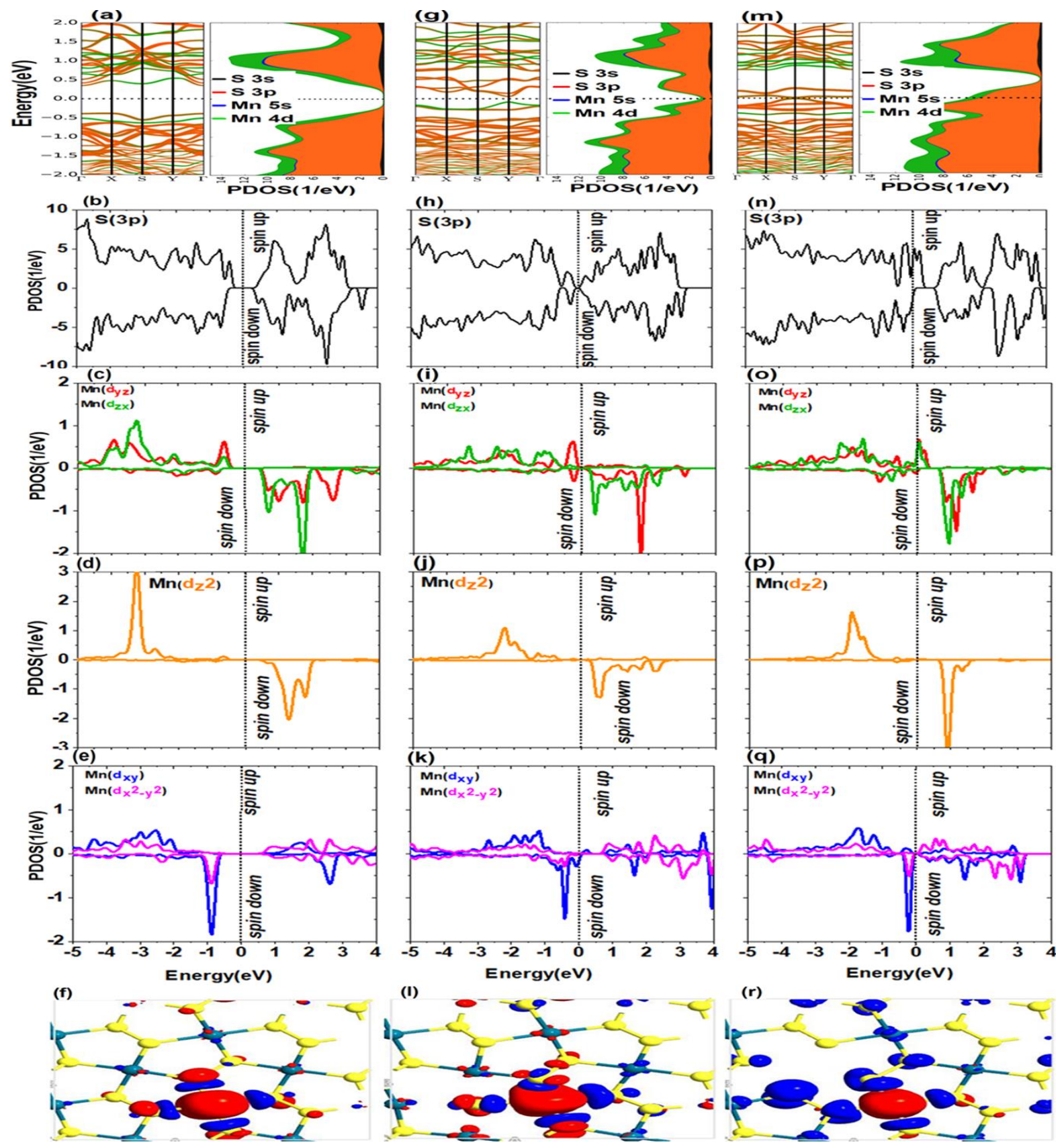

Fig. 3. (a-f) band structure and DOS of $p$ and $d$ orbitals and spin density difference in the presence of transition metal impurity $(\mathrm{Mn}),(\mathrm{g}-\mathrm{l})$ in the presence of the $S$ atom vacancy defect, and $(\mathbf{m}-\mathbf{r})$ in the presence of the Pd atom vacancy defect.

Then we investigate the effect of removing a Pd atom, position No.2 in Fig. 1(r). By removing this atom, four first-neighbor $\mathrm{S}$ atoms of $\mathrm{Pd}$ will have free bonds. The magnetic energy $\left(\Delta \mathrm{E}_{\text {spin }}\right)$ arising from the $\mathrm{Pd}$ vacancy in the presence of $\mathrm{Mn}$ is equal to $501.11 \mathrm{meV}$ as demonstrated in Table S5. In other words, the magnetic energy of $\mathrm{Pd}_{2} \mathrm{~S}_{4}$ is also reduced relative to the initial and $\mathrm{S}$ vacancy states. The intensity of magnetization has also been mitigated by $0.472 \mu_{B}$ relative to the case without vacancy defect. The $S$ 
atom, which is in commonly bonds with $\mathrm{Mn}$ and $\mathrm{Pd}$ atoms, should gain more electrons from $\mathrm{Mn}$ by removing $\mathrm{Pd}$. In this case, the magnetic moment is also reduced by $0.472 \mu_{B}$, which means that the magnetic moment of adjacent $S$ atoms increases, as presented in Table S6. It can also be found from spin density shown in Fig. 3(r). The accurate value of this increase is presented in Tables S2 and S6 in the SI. According to Fig. $3(\mathrm{~m})$, which shows the band structure in the presence of $\mathrm{Mn}$ impurity and Pd vacancy, and Table S7 in the SI, there is no gap energy in this case. As shown in the $\mathrm{SI}$, Figs. S2 and S3, 3d orbitals of $\mathrm{Mn}$ atom and $3 p$ orbitals of $\mathrm{S}$ atom cross the Fermi level. Regarding Fig. $3(n)$, the $3 p$ orbital of $S$ atom and $\mathrm{d}_{\mathrm{yz}}$ and $\mathrm{d}_{\mathrm{zx}}$ (Fig. 3(o))orbitals of $\mathrm{Mn}$ play crucial roles in cancelling the $0.73 \mathrm{eV}$ gap energy when only $\mathrm{Mn}$ atom is present as an impurity in the structure.

The last figures of Figs. 3(i) and 3(r), illustrate the difference of spin density in the presence of S and Pd vacancies, respectively, where the $\mathrm{Mn}$ doping in both of the cases causes the extension of polarity from the impurity atom to neighboring atoms. This polarization is more obvious when the $\mathrm{Pd}$ atom is removed from the system. Electrons of the $3 p$ orbital of the nearest $S$ atom have an opposite spin relative to electrons of the $3 \mathrm{~d}$ orbital of the $\mathrm{Mn}$ atom.

In the following, the effect of adding of $V$ element to $\mathrm{Pd}_{2} \mathrm{~S}_{4}$ structure is examined, which have a moderate intensity of magnetization. When $V$ atom with a valance configuration of $4 \mathrm{~s}^{2} 3 \mathrm{~d}^{3}$ is substituted for a $\mathrm{Pd}$ atom in the structure, must lose electrons as much as Pd atoms. According to Table $\mathrm{S} 7$ in the $\mathrm{SI}$, in the presence of $\mathrm{V}$ atom the gap energy of doped $\mathrm{Pd}_{2} \mathrm{~S}_{4}$ is $0.44 \mathrm{eV}$ which is decreased nearly by $0.76 \mathrm{eV}$ with respect to the pure system. As shown in Fig. 4(a-e), 3d orbitals of the $V$ atom and $3 p$ orbitals of the $S$ atom have an excellent hybrid in the valence and conduction bands. The contributions of low-spin and highspin orbitals are entirely specified in valence and conduction bands, respectively. Also, VBM entirely coincides with $\Gamma$ point, and $C B M$ lies on point $S$ in the first Brillouin zone. According to data presented in Fig. 2(a), the intensity of magnetization of $\mathrm{V}$ is $1.395 \mu_{B}$ when there are no $\mathrm{S}$ and $\mathrm{Pd}$ vacancy defects. As can be seen, this value is considerably lower than the first considered group. The $3 p$ orbital of the $S$ atom has no contribution in the magnetization of the structure thus the DOS diagram for high-spin and low-spin states is almost symmetrical. Therefore, in this case, the major part of magnetization is due to the $3 \mathrm{~d}$ orbital of V. Fig. 2(c) clearly indicates that exchange interaction and, consequently, splitting of high and low spins of $\mathrm{V}^{+4}$ are lower than $\mathrm{Mn}^{+4}$. In this case, the maximum spin states separation is related to $\mathrm{d}_{\mathrm{z}^{2}}$ (Fig. 4(d))as about $3 \mathrm{eV}$. Moreover, one of the spin states is full, and the other is entirely vacant. Thus, a major part of $1.39 \mu_{B}$ is related to this orbital. The residual value of magnetization intensity, equivalent to $0.39 \mu_{B}$, is attributed to other orbitals. Therefore, due to the spin splitting, the primary perception that the magnetization of $\mathrm{V}$ atom is equal to the missed four electrons one cannot be true. The main point in doping with $\mathrm{V}$ and Co (according to Fig. 2(c)) is the considerable contribution of $\mathrm{d}_{\mathrm{z}^{2}}$ in the magnetization. In contrast to the relatively large contributions of three orbitals $\mathrm{d}_{\mathrm{z}^{2}}, \mathrm{~d}_{\mathrm{yz}}$, and $\mathrm{d}_{\mathrm{zx}}$ (Fig. 2) in magnetization of the first group ( $\mathrm{Fe}, \mathrm{Mn}$, and $\mathrm{Cr}$ ), the major contribution for the second group is related to $\mathrm{d}_{\mathrm{z}^{2}}$ orbital . $V$ and Co have 5 and 9 electrons in their last energy level, respectively. Therefore, after being involved in the $\mathrm{Pd}_{2} \mathrm{~S}_{4}$ structure, they lose four electrons and will have one and five electrons in the $3 \mathrm{~d}$ energy level. Hence, the single electron of $\mathrm{V}$ is placed in high-spin $\mathrm{d}_{\mathrm{z}^{2}}$ orbital, which is why it has the most percentage of magnetization. Also, four electrons of Co with opposite spins occupy two orbitals of $\mathrm{d}_{\mathrm{yz}}$ and $\mathrm{d}_{\mathrm{zx}}$, and only the remaining one electron goes to $\mathrm{d}_{\mathrm{z}^{2}}$ orbital. There is no electron for Ti of valence shell $4 \mathrm{~s}^{2} 3 \mathrm{~d}^{2}$ after being involved in the $\mathrm{Pd}_{2} \mathrm{~S}_{4}$ structure. 

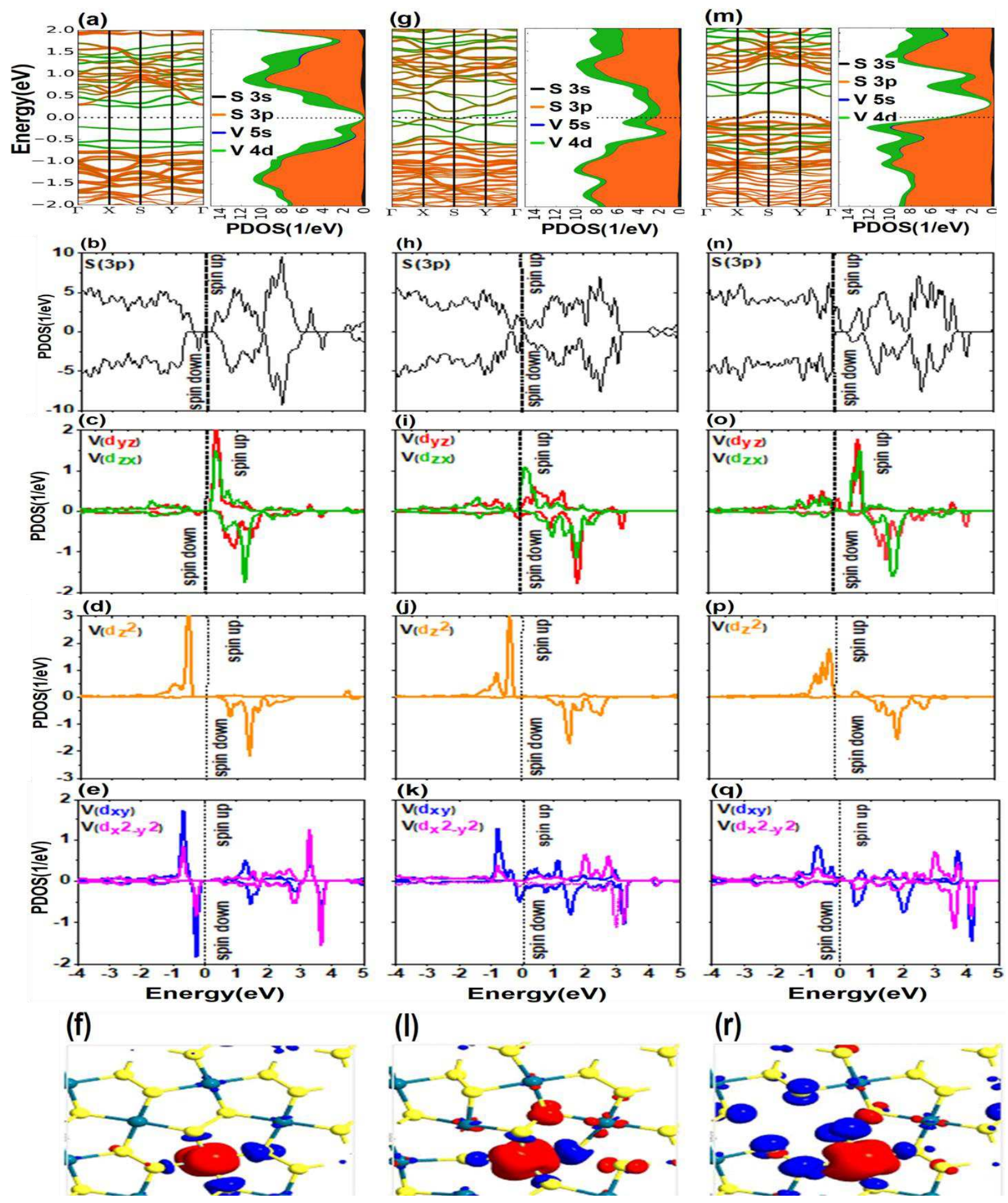

Figure 4. (a-f) band structure and DOS of $p$ and $d$ orbitals and spin density difference in the presence of transition metal impurity (V), (g-i) in the presence of the $S$ atom defect, and $(m-r)$ in the presence of the Pd atom defect. 
According to Figs. $\mathrm{S} 2$ and $\mathrm{S} 3$ and Table $\mathrm{S} 7$ in the $\mathrm{SI}$, the $\mathrm{Pd}_{2} \mathrm{~S}_{4}$ structure becomes conductive because of $\mathrm{S}$ vacancy formation in the first neighbor of the doped structure. It can be found from Figs. S2 and S3 that Fermi level interrupts the $3 d$ orbital of $V$ and $3 p$ orbital of $S$. Thus, $3 d$ and $3 p$ orbitals with high and low spins not only cross Fermi level but also interrupt each other at $\mathrm{S}$ point in the band structure due to the $\mathrm{S}$ vacancy defect. As illustrated in Fig. $4(\mathrm{~g}-\mathrm{i})$ and Tables $\mathrm{S} 2$ and $\mathrm{S} 4$ of the $\mathrm{SI}$, the vacancy defect of S atom in the first neighborhood of $\mathrm{V}$-doped $\mathrm{Pd}_{2} \mathrm{~S}_{4}$ can increase the intensity of magnetization to $1.946 \mu_{B}$ compared to the no vacancy one which is $1.39 \mu_{B}$, where like the previous case, $3 p$ (Fig. $4(\mathrm{~h})$ ) orbitals have fairly symmetrical shapes and no magnetization. In this case, the significant contribution of $\mathrm{d}_{\mathrm{z}^{2}}$ (Fig. $4(j))$ to magnetization is clear. The high spin of $\mathrm{d}_{\mathrm{z}^{2}}$ (Fig. 4(j)) stays on the valence band, while this orbital has a vacant opposite spin. Also, the energy difference of high-spin and low-spin states under the effect of exchange interaction in $\mathrm{d}_{\mathrm{z}^{2}}$ is about $2.5 \mathrm{eV}$. For the remainder of magnetization, the other $3 \mathrm{~d}$ orbitals must be considered. In the presence of vacancy defect at the first neighborhood (as shown in Fig. 2(c)), other orbitals have approximately the same contribution to magnetization. According to Table S5 in the $\mathrm{SI}$, the spin energy arising from the Pd atom vacancy defect in the V-dopped structure is $-432.72 \mathrm{meV}$. Also, based on Fig. 2(a), the magnetic moment due to the Pd vacancy defect has increased about $0.70 \mu_{B}$ compared to the pure system.

Regarding Figs. 2(c) and $4(k), d_{x^{2}-y^{2}}$ and $d_{x y}$ have made a profound difference with three other orbitals $\mathrm{d}_{\mathrm{z}^{2}}, \mathrm{~d}_{\mathrm{yz}}$, and $\mathrm{d}_{\mathrm{zx}}$ in terms of spin separation. Therefore, the main effect of increasing the magnetic moment compared to the pure system can be due to the effect of the new crystal field on these orbitals. Figs. S2 and S3 and the magnitude of the gap energy presented in Table S7 in SI show that the structure becomes conductive because of Pd atomic vacancy so that Fermi level shifts to the within of 3p Fig. 4(mn) and 3d Fig. $4(m, o, p, q)$ orbitals. In the last figures of each column of Fig. $4(f, l, r)$, the spin density at impurity atom $(V)$ has been illustrated for the structure without and with vacancies. The behavior of atoms adjacent to impurity in considered cases is like the behavior of the atoms adjacent to the Mn impurity (see Fig.3). The main difference is the lower intensity of magnetization in the presence of $\mathrm{V}$, which the reasons were investigated above.

When the Ni metal with valence shell of $4 \mathrm{~S}^{2} 3 \mathrm{~d}^{8}$ involves in the studied structure as the representation of the third group ( $\mathrm{Sc}, \mathrm{Ni}, \mathrm{Cu}$, and $\mathrm{Zn}$ ), it shows no magnetic properties. The $\mathrm{Ni}$ atom has three more electrons than $\mathrm{Mn}$, and in a straightforward comparison with the $\mathrm{Mn}$ atom, it is expected that these additional electrons can cover the entire magnetic moment obtained in the presence of $\mathrm{Mn}$.

As indicated in Fig. 2, Ni doping does not influence the magnetic properties of the intended system. The DOS diagrams for high-spin and low-spin states are perfectly symmetrical and neutralize the magnetic effects of each other. From Fig. $5(a-f)$, it is observed that $d_{z^{2}}$ (Fig. $\left.5(d)\right) d_{y z}$, and $d_{z x}$ (Fig. 5(c)) are within the valence band and wholly occupied. Therefore, electrons of $\mathrm{Ni}^{+4}$ occupy orbitals of the lowest energy pairwise with opposite spins in a pair. Hence, there occurs no magnetization in this structure, and the magnetic contribution of every component is zero. The role of $\mathrm{Ni}$ is similar to $\mathrm{Pd}$ because both of them have no spin separation and no magnetizing effect on the system. Similarly, Sc, $\mathrm{Cu}$, and $\mathrm{Zn}$ also have symmetrical high and low spins densities and no spin separation. In this case, the required field for $d$ orbitals splitting is the crystal field, which does not compete with exchange interaction for separating high-spin and low-spin states. Table S7 in the SI demonstrates that the gap energy variation in the presence of $\mathrm{Ni}$ is less than in the presence of Mn and V. According to Figs. S2 and S3 in SI, it can be stated that the structure still remains semi-conductive when $\mathrm{Ni}$ is introduced, and the type of gap energy remains indirect.

VBM and CBM are coincident with $\Gamma$ point. The $S$ vacancy in this structure leads to the band-gap alteration but the system is non-magnetic (see Fig. $5(\mathrm{~h}, \mathrm{i}, \mathrm{j}, \mathrm{k}, \mathrm{I})$ ). In Table $\mathrm{S} 7$ of the $\mathrm{SI}$, the gap energy values arising from this defect are presented. From Figs. 5(g), Fig. S2, and Fig. S3, it is found that the band-gap is direct 
and VBM and CBM coincide with $X$ point. The structure still remains nonmagnetic (see Figs. 5(n, o, p, q, r)) by removing the $\mathrm{Pd}$ atom and in the absence of exchange interaction, leading to the separation of spin states. In this case, the main difference is the intersection of Fermi level by $p$ orbitals of $S$ and $d_{y z}$ and $d_{z x}$ (Fig. $5(\mathrm{~m}, \mathrm{o})$ ) orbitals of Pd. The addition of free electrons because of the $\mathrm{S}$ atom and initial symmetry elimination can be the reason for this behavior of energy level in altering the band-gap in states with vacancy.
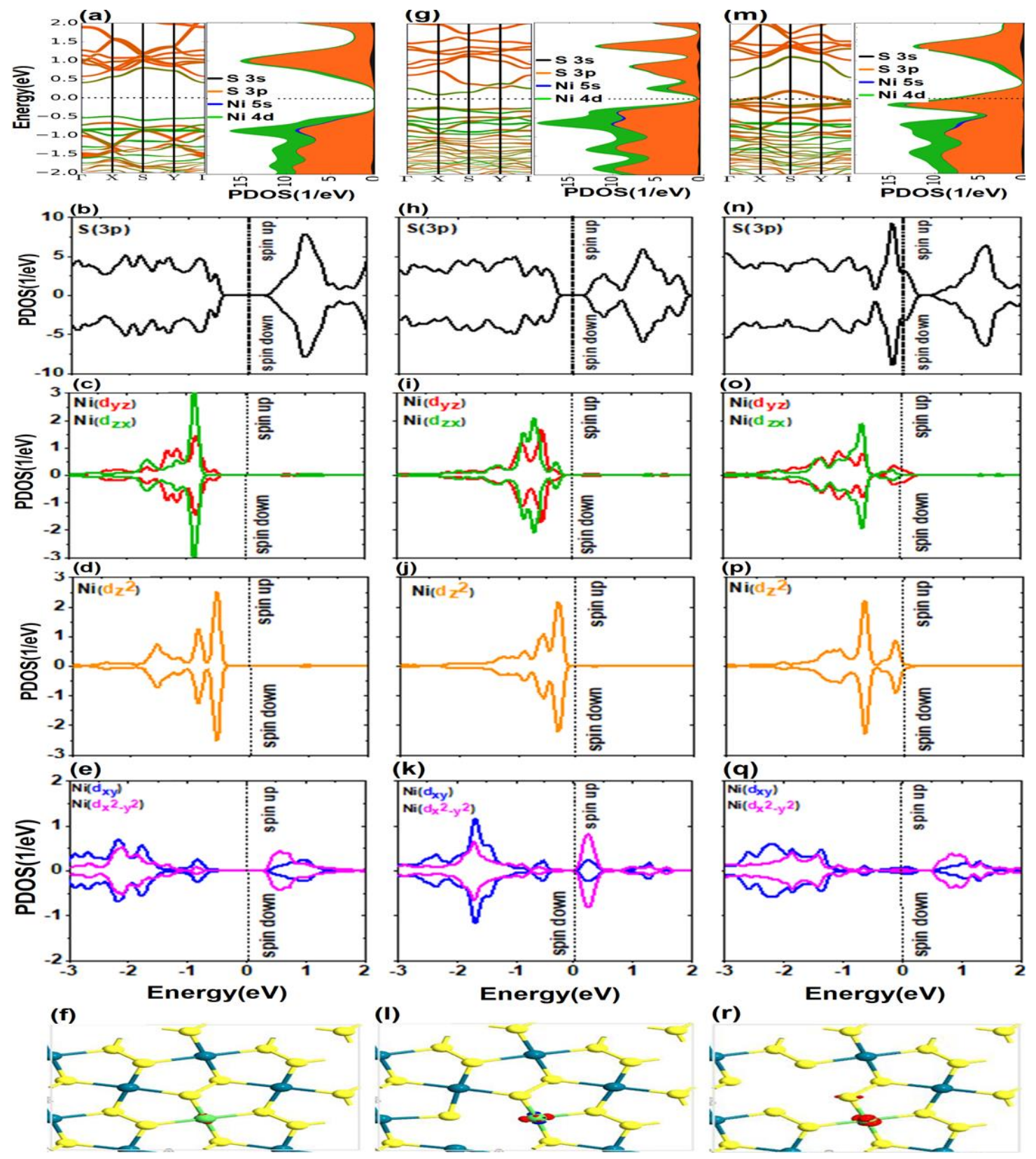

( $r)$

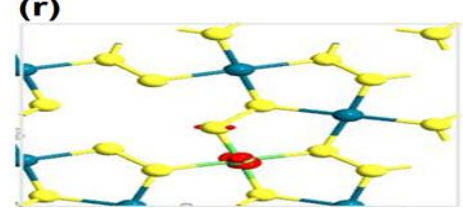

Figure 5. (a-f) bandstructure and PDOS of $p$ and $d$ orbitals and spin density difference in the presence of transition metal impurity ( $\mathrm{Ni}),(\mathbf{g}-\mathbf{i})$ in the presence of the $\mathrm{S}$ atom defect, and $(\mathbf{m}-\mathbf{r})$ in the presence of the $\mathrm{Pd}$ atom defect. 


\section{Conclusion}

This paper studied the development of magnetic properties and the electronic properties of monolayer $\mathrm{Pd}_{2} \mathrm{~S}_{4}$ under the effect of doping with $3 \mathrm{~d}$ transition metals with/without vacancy defects of $\mathrm{S}$ and $\mathrm{Pd}$ atoms. The crystal field decreases the degeneracy of $d$ orbitals of $3 d T M$, and these orbitals are split approximately based on the square planar model. The addition of exchange interaction in the presence of $3 d$ transition metals causes the new spin separations, especially in $d$ orbitals. The spin splitting of $d_{z^{2}}$ is sometimes more considerable and effective in establishing the magnetic moment of the system where the spin energy difference of $\mathrm{d}_{\mathrm{z}^{2}}$ between high and low spins for Mn doping is almost $4 \mathrm{eV}$. This separation causes one of the spin states to be full and another one to be vacant. Calculations for all $3 \mathrm{~d}$ TM of the periodic table indicate that doping with $\mathrm{Mn}, \mathrm{Cr}$, and Fe leads to the maximum intensity of magnetization in the structure. Some of $3 \mathrm{~d}$ metals, such as $\mathrm{Ti}, \mathrm{V}$, and $\mathrm{Co}$, cause less magnetization in the structure. Finally, doping with the $3 \mathrm{~d}$ metals such as $\mathrm{Sc}, \mathrm{Ni}, \mathrm{Cu}$, and $\mathrm{Zn}$ atoms, results in no magnetic properties in the system. By applying the $\mathrm{S}$ and $\mathrm{Pd}$ atomic vacancies which are next to the TM impurities, the system symmetry mitigates, and the number of free electrons, which are previously in a bond with other atoms, increases. Therefore, the atomic vacancies, as obtained here, has no significant effect on the magnetic properties of the system and can directly affect the electronic properties such as the gap energy.

\section{Data availability}

All data generated or analyzed during this study are included in this published article (and its Supplementary Information flies).

\section{References}

1. Geim. A, Novoselov. K. The rise of graphene. Nanoscience and Technology pp. 11-19; https://doi.org/10.1142/9789814287005 0002 (2009).

2. Jin. Ch, Lin. F, Suenaga. K, lijima. S. Fabrication of a Freestanding Boron Nitride Single Layer and Its Defect Assignment. Phys.Rev. Lett 102, 195505; https://doi.org/10.1103/PhysRevLett.102.195505 (2009).

3. Son. Y, Cohen. M, Louie. S. Energy Gaps in Graphene Nanoribbons. Phys. Rev. Lett 97, 216803; https://doi.org/10.1103/PhysRevLett.97.216803 (2006).

4. Kang. D., et al. High-Performance Transition Metal Dichalcogenide Photodetectors Enhanced by Self-Assembled Monolayer Doping. Adv. Funct. Mater 25, 4219-4227; https://doi.org/10.1002/adfm.202109794 (2015).

5. Chang. M, Fan. H, Chowdhury. M, Sawatzky. G, Nojeh. A. Heat localization through reduced dimensionality. Phys. Rev. B 98, 155422; https://doi.org/10.1103/PhysRevB.98.155422 (2018).

6. Gibertini. M, Koperski. M, Morpurgo. A, Novoselov. K. Magnetic 2D materials and heterostructures. Nature Nanotechnology 14, 408-419; https://doi.org/10.1038/s41565-0190438-6 (2019).

7. S.Burch. K, Mandrus. D, Park. J. Magnetism in two-dimensional van der Waals materials. Nature 563, 47-52; https://doi.org/10.1038/s41586-018-0631-z (2018).

8. Chen. W, et al., Direct observation of van der Waals stacking-dependent interlayer magnetism. SCIENCE 366, 983-987; https://doi.org/10.1126/science.aav1937 (2019). 
9. Ma. Y, et al., Evidence of the Existence of Magnetism in Pristine VX2 Monolayers $(X=S, S e)$ and Their Strain-Induced Tunable Magnetic Properties. ACS Nano 6, 2, 1695-1701; https://doi.org/10.1021/nn204667z (2012).

10. Deng. Y, et al., Gate-tunable room-temperature ferromagnetism in two-dimensional Fe3GeTe2. Nature 563, 94-99; https://doi.org/10.1038/s41586-018-0626-9 (2018).

11. Lee. J, et al. Ising-Type Magnetic Ordering in Atomically Thin FePS3. Nano Lett 16, 12, $7433-$ 7438; https://doi.org/10.1021/acs.nanolett.6b03052 (2016).

12. O'Hara. DJ, et al., Room Temperature Intrinsic Ferromagnetism in Epitaxial Manganese Selenide Films in the Monolayer Limit. Nano Lett 5, 3125-3131; https://doi.org/10.1021/acs.nanolett.8b00683 (2018).

13. Sethulakshmi. N, et al., Magnetism in two-dimensional materials beyond graphene. Materials Today 27, 107-122; https://doi.org/10.1016/i.mattod.2019.03.015 (2019).

14. Yun. WS, Han. SW, Hong. S C, Kim. IG, Lee .JD. Thickness and strain effects on electronic structures of transition metal dichalcogenides: $2 \mathrm{H}-\mathrm{MX} 2$ semiconductors ( $\mathrm{M}=\mathrm{Mo}, \mathrm{W} ; \mathrm{X}=\mathrm{S}$, Se, Te). Phys. Rev. B 85, 033305; https://doi.org/10.1103/PhysRevB.85.033305 (2012).

15. Lin. $Z$, , et al., Defect engineering of two-dimensional transition metal dichalcogenides. 2D Mater 3, 022002; https://doi.org/10.1088/2053-1583/3/2/022002 (2016).

16. Acta. C, Sahin. H, Giraci. S. Stable, Single-Layer MX2 Transition-Metal Oxides and Dichalcogenides in a Honeycomb-Like Structure. J. Phys. Chem. C 116, 16, 8983-8999; https://doi.org/10.1021/ip212558p (2012).

17. Yeh. CH. Computational Study of Janus Transition Metal Dichalcogenide Monolayers for Acetone Gas Sensing. ACS Omega 48, 31398-31406; http://dx.doi.org/10.1021/acsomega.0c04938 (2020).

18. Villaos. RAB, et al., Evolution of the Electronic Properties of $\mathrm{ZrX2}(\mathrm{X}=\mathrm{S}$, Se, or Te) Thin Films under Varying Thickness. J. Phys. Chem. C 125, 1, 1134-1142; https://doi.org/10.1021/acs.jpcc.0c10085 (2021).

19. Jiang. $X$, et al., Recent progress on 2D magnets: Fundamental mechanism, structural design and modification. Applied Physics Reviews 8, 031305; https://doi.org/10.1063/5.0039979 (2021).

20. Wang. $Y$, et al., Defects engineering induced room temperature ferromagnetism in transition metal doped MoS2. Materials \& Design 121, 77-84; https://doi.org/10.1016/j.matdes.2017.02.037 (2017).

21. Lv.HY, Lu. W J, Shao .DF, Liu. Y, Sun. YP. Strain-controlled switch between ferromagnetism and antiferromagnetism in $1 \mathrm{~T}-\mathrm{CrX} 2$ (X=Se, Te) monolayers. Phys. Rev. B 92, 214419; https://doi.org/10.1103/PhysRevB.92.214419 (2015).

22. Avsar. A, et al., Defect induced, layer-modulated magnetism in ultrathin metallic PtSe 2. Nat Nanotechnol 7, 674-678; https://doi.org/10.1038/s41565-019-0467-1 (2019).

23. Ma. Y, et al., Electronic and magnetic properties of perfect, vacancy-doped, and nonmetal adsorbed MoSe2, MoTe2 and WS2 monolayers. Phys. Chem. Chem. Phys 13, 15546-15553; https://doi.org/10.1039/C1CP21159E (2011).

24. Ataca. C, Ciraci. S. Functionalization of Single-Layer MoS2 Honeycomb Structures. Phys. Chem. C 115, 27, 13303-13311; https://doi.org/10.1021/jp2000442 (2011).

25. Zhang. J, Zheng. H, Han. R, Du. X, Yan. Y. Tuning magnetic properties of CrS2 monolayer by doping transition metal and alkaline-earth atoms. Journal of Alloys and Compounds 647, 75-81; https://doi.org/10.1016/j.jallcom.2015.05.175 (2015).

26. Yue. Q, Chang. Sh, Qin. Sh, Li. J. Functionalization of monolayer MoS2 by substitutional doping: A first-principles study. Physics Letters A 377, 1362-1367; https://doi.org/10.1016/i.physleta.2013.03.034 (2013). 
27. Tedstone. AA, Lewis. DJ, OBrien. P. Synthesis, Properties, and Applications of Transition MetalDoped Layered Transition Metal Dichalcogenide. Chem. Mater 28, 7, 1965-1974; https://doi.org/10.1021/acs.chemmater.6b00430 (2016).

28. Yang. B, Zheng. H, Han. R, Du. X, Yan. Y. Tuning the magnetism of a ZrS2 monolayer by substitutional doping. RSC Adv 4, 54335-54343; https://doi.org/10.1039/C4RA08513B (2014).

29. Hashemi. D, Lizuka. H. Substitutional $4 d$ transition metal doping in atomically thin lead. RSC $A d v$ 11, 6182-6187; https://doi.org/10.1039/DORA09742J (2021).

30. Zhang. Y, Zhaho. Y, Xu. Y, He. L. Tuning magnetic and optical properties of monolayer WSe2 by doping C, N, P, O, S, F, and Cl: First principles study. Solid State Communications 327, 114233; http://dx.doi.org/10.1016/j.ssc.2021.114233 (2021).

31. Qu. Y. et al., Pentagonal transition-metal (group X) chalcogenide monolayers: Intrinsic semiconductors for photocatalysis. International Journal of Hydrogen Energy 46, 9371-9379; https://doi.org/10.1016/j.ijhydene.2020.12.085 (2021).

32. Ahmad. S, Schreckenbach. G. Ab initio study of strain and electric field dependent variation in electronic and thermoelectric properties of PdS2. Materials today communications 24, 100976; https://doi.org/10.1016/j.mtcomm.2020.100976 (2020).

33. Zhao. B. 2D Metallic Transition-Metal Dichalcogenides: Structures, Synthesis, Properties, and Applications. Adv. Funct. Mater. 2105132; https://doi.org/10.1002/adfm.202105132 (2021).

34. Chen. E, Xu. W, Chen. J, Warner. J.H. 2D layered noble metal dichalcogenides (Pt, Pd, Se, S) for electronics and energy applications. Materials Today Advances 7, 100076; https://doi.org/10.1016/j.mtadv.2020.100076 (2020).

35. Feng. L-Y, et al., Layer-dependent band engineering of Pd dichalcogenides: a first-principles study. New J. Phys, 22, 053010; https://orcid.org/0000-0003-0351-4253 (2020).

36. Gan. Y. et al. Structural and electronic properties of PdS2 nanoribbons. Journal of Magnetism and Magnetic Materials 458, 310-316; https://doi.org/10.1016/i.jmmm.2018.03.044 (2018).

37. A.Moujaes. E, A. Diery. W. Optical properties and stability of new two-dimensional allotropes of PdS2, PdSe2 and PdSSe monolayers. Physica E: Low-dimensional Systems and Nanostructures 128, 114611; https://doi.org/10.1016/j.physe.2020.114611 (2021).

38. Saraf. D, Chakraborty. S, Kshirsagar. A, Ahuja. R. In pursuit of bifunctional catalytic activity in PdS2 pseudo-monolayer through reaction coordinate mapping. Nano Energy 49, 283-289; https://doi.org/10.1016/j.nanoen.2018.04.019 (2018).

39. B.Isaacs. E, Wolerton. Ch. Inverse Band Structure Design via Materials Database Screening: Application to Square Planar Thermoelectrics. Chem. Mater 30, 5, 1540-1546; https://doi.org/10.1021/acs.chemmater.7b04496 (2018).

40. J. P. Perdew, K. Burke and M. Ernzerhof, Phys. Rev. Lett 77, 3865-3868; https://doi.org/10.1103/PhysRevLett.77.3865 (1996)

41. P. E. Blöchl, Phys. Rev. B 50, 17953-17979; https://doi.org/10.1103/PhysRevB.50.17953 (1994).

42. J. Phys. Cond. Matt. 14, 2745 (2002). https://iopscience.iop.org/article/10.1088/09538984/14/11/302/meta.

43. Naghavi. S.S, He. J, Xia. Y, Wolerton. Ch. Pd2Se3 Monolayer: A Promising Two-Dimensional Thermoelectric Material with Ultralow Lattice Thermal Conductivity and High Power Factor. Chem. Mater 30, 16, 5639-5647; https://doi.org/10.1021/acs.chemmater.8b01914 (2018).

\section{Author contributions}

M.G. contributed to implementation of the research and the analysis of the results and performed the numerical simulations. M.G. and H.R.S. and Z.G developed the theoretical formalism, performed the 
analytic calculations. M.G. and Z.G. wrote the main manuscript text. All authors discussed the results and contributed to the final manuscript. H.R.S. supervised the project. 


\section{Supplementary Files}

This is a list of supplementary files associated with this preprint. Click to download.

- Supplementary.pdf 